Tjalling C. Koopmans Research Institute Trlligh Koopmen

Discussion Paper Series nr: 11-18

\title{
Expected optimal feedback with Time-Varying Parameters
}

Marco P. Tucci

David A. Kendrick

Hans M. Amman 


\section{Tjalling C. Koopmans Research Institute Utrecht School of Economics Utrecht University}

Kriekenpitplein 21-22

3584 TC Utrecht

The Netherlands

telephone $\quad+31302539800$

fax +31302537373

website www.koopmansinstitute.uu.nl

The Tjalling C. Koopmans Institute is the research institute and research school of Utrecht School of Economics.

It was founded in 2003, and named after Professor Tjalling C. Koopmans, Dutch-born Nobel Prize laureate in economics of 1975.

In the discussion papers series the Koopmans Institute publishes results of ongoing research for early dissemination of research results, and to enhance discussion with colleagues.

Please send any comments and suggestions on the Koopmans institute, or this series to L.M.vanDort@uu.nl

ontwerp voorblad: WRIK Utrecht

\section{How to reach the authors}

Please direct all correspondence to the first author.

Marco P. Tucci

Dipartimento di Economia Politica

Universitá di Siena

Piazza S. Francesco 7

53100 Siena, Italy

E-mail: tucci@unisi.it

David A. Kendrick

Department of Economics

University of Texas

Austin, Texas 78712

USA

E-mail: kendrick@austin.utexas.edu

Hans M. Amman

Utrecht School of Economics

Utrecht University

Kriekenpitplein 21-22

3584 TC Utrecht

Netherlands

E-mail: h.m.amman@uu.nl 
Utrecht School of Economics

Tjalling C. Koopmans Research Institute

Discussion Paper Series 11-18

\title{
Expected optimal feedback with Time-Varying Parameters
}

\author{
Marco P. Tucci \\ David A. Kendrick \\ Hans M. Ammanc \\ ${ }^{a}$ Dipartimento di Economia Politica \\ Universitá di Siena \\ ${ }^{b}$ Department of Economics \\ University of Texas \\ 'Utrecht School of Economics \\ Utrecht University \\ October 2011
}

\begin{abstract}
In this paper we derive the closed loop form of the Expected Optimal Feedback rule, sometimes called passive learning stochastic control, with time varying parameters. As such this paper extends the work of Kendrick (1981,2002, Chapter 6) where parameters are assumed to vary randomly around a known constant mean. Furthermore, we show that the cautionary myopic rule in Beck and Wieland (2002) model, a test bed for comparing various stochastic optimizations approaches, can be cast into this framework and can be treated as a special case of this solution.
\end{abstract}

Keywords: Optimal experimentation, stochastic optimization, time-varying parameters, expected optimal feedback

JEL classification: C63, E61

\section{Acknowledgements}

We would like to thank Michael Reiter for his careful discussion of our paper at the workshop in honor of Steve Turnovsky, held in Vienna May 20-22, 2010.

Furthermore, we received helpful comments from some of the other participants and an anonymous referee. 


\section{Introduction}

Most of the times, if not always, the use of optimal control in Economics is associated to systems with unknown parameters. For this reason a lot of attention has been paid to the appropriate use of their estimates in the design of optimal policies since the early seventies (see, e.g., Chow (1973, 1975a), Kendrick and Majors (1974), Turnovsky $(1975,1976,1977))$. In many cases, for convenience sake, the economic systems are assumed linear, or linearized versions of non-linear models, and the true unknown econometric parameters constant through time. In order to find a control rule making an optimal use of both the estimated parameters and the associated covariance matrix, the system equations of the control model have been sometimes rewritten as having random coefficients (see, e.g. Chow (1975b), Kendrick (1981, 2002, Chapter 6)). Only in a few instances the true unkownon parameters of the linear system have been assumed time-varying (see, e.g. Kendrick (1981, 2002, Chapter 10), Tucci (1989, 1997, 2004)).

A recent result in Granger (2008, page 4.) indeed may make the combination of linear systems and time-varying parameters even more promising. As shown in his Whites Theorem any non-linear model can be approximated by a time-varying parameter linear model. And, the goodness of approximation depends upon how smoothly parameters change over time (Granger (2008, page 5.)). Then controlling a linear system where parameters are allowed to vary, either in a parametric way or in a way based on Kalman Filter, can be viewed as an approximation to controlling the associated nonlinear model.

The introduction of stochastic parameters in a control theory framework frequently leads to the use of approximations of the dynamic programming algorithm. For this reason researchers in the control field are often induced to discuss in great details the approximation of their choice and treat implicitly the alternative methods. For instance, Kendrick (1981, 2002) and Tucci $(1989,1997,2004)$ discuss at length the DUAL algorithm, but they fail to spell out the effects of the introduction of system equations with timevarying parameters on the computation of the familiar expected optimal feedback control, i.e. the case where decision makers ignore future learning about the unknown parameters in the design of the current policy. A relevant exception is represented by Beck and Wieland (2002) that assume a system with one unknown parameter following a random walk. This paper aims to fill the gap for the general case of several time-varying parameters, either stationary or non-stationary. It is therefore an extension of Kendrick (1981, 2002, Chapter 6) which provides a similar derivation for models with 
parameters assumed to vary randomly around a constant mean. ${ }^{1}$

In Section 2 of this paper the problem is stated in terms of a linear system with time-varying parameters and a quadratic objective function. Then the approximate optimal expected cost for periods $N, N-1$ and a generic period $j$ are derived. The approximation is based on the information on the time-varying parameters available at the beginning of the planning horizon, that is time 0. This is similar, in spirit, to the assumption used in Chow (1975a, Chapter 10) and boils down to ignoring possible revisions of the density of the stochastic parameters by observations on the states and controls in the planning horizon, i.e. periods 1 through $\mathrm{N}$. Then, as argued in Chow (1975a, page 223), if the information on the parameters available at time 0 is large compared to the additional knowledge that can be acquired during the planning horizon, as is usually the case when an econometric model is used for policy analysis, the approximation of these pages is likely to be close to the true unknown optimal. In any case, this approximation represents "an upper bound to the loss arising from uncertainty in the parameters." 2

It is worthwhile to point out that the formulae associated with the timevarying parameters problem look exactly the same as those in Kendrick (1981, 2002, Chapter 6), except for the fact that now the expectation on the stochastic parameters is conditional on the information available at time 0 , thus $E_{0}$. Section 6 shows that this minor notational difference has substantial computational consequences. In section 7. the Beck and Wieland (2002) model is cast in the framework of this paper.

It is observed that for the parameter set used in Amman et al. (2008) and Beck and Wieland (2002) the approximated expected optimal feedback control derived in these pages is indeed the expected optimal feedback control, because the associated feedback matrices are independent of the future path of the time-varying parameters. This is the so-called myopic cautionary rule in Beck and Wieland (2002, page 1365). The approximation presented here can be viewed also as a generalization of the myopic cautionary rule. Finally, it may prove very useful, as a benchmark, in assessing the ex-post performance of more sophisticated methods involving active learning, such as DUAL in Kendrick $(1981,2002)$ and Tucci $(1997,2004)$ or the dynamically optimal control rule in Beck and Wieland (2002).

\footnotetext{
${ }^{1}$ In a discussion paper Amman and Kendrick (2001) consider the case where a subset of parameters is stochastic and follows a first order Markov process with a time-varying transition matrix, D, and covariance matrix. They suggest finding the EOF control using an augmented state vector including both the states and the stochastic parameters. This paper suggests an alternative approach to solve the same problem.

${ }^{2}$ See Chow (1975a, page 223).
} 


\section{Statement of the Problem}

A general quadratic linear control model can be stated as follows: select the control vectors $\mathbf{u}_{0}, \ldots, \mathbf{u}_{N-1}$ to minimize the criterion functional

$$
J=E\left\{C_{N}\right\}=E\left\{L_{N}\left(\mathbf{x}_{N}\right)+\sum_{k=0}^{N-1} L_{k}\left(\mathbf{x}_{k}, \mathbf{u}_{k}\right)\right\}
$$

with $E$ the expectation operator and with,

$$
L_{N}\left(\mathbf{x}_{N}\right)=\frac{1}{2} \mathbf{x}_{N}^{\prime} \mathbf{W}_{N} \mathbf{x}_{N}+\mathbf{w}_{N}^{\prime} \mathbf{x}_{N}
$$

and

$$
L_{k}\left(\mathbf{x}_{k}, \mathbf{u}_{k}\right)=\frac{1}{2} \mathbf{x}_{k}^{\prime} \mathbf{W}_{k} \mathbf{x}_{k}+\mathbf{w}_{k}^{\prime} \mathbf{x}_{k}+\mathbf{x}_{k}^{\prime} \mathbf{F}_{k} \mathbf{u}_{k}+\frac{1}{2} \mathbf{u}_{k}^{\prime} \boldsymbol{\Lambda}_{k} \mathbf{u}_{k}+\lambda_{k}^{\prime} \mathbf{u}_{k}
$$

subject to the system equations

$$
\mathbf{x}_{k+1}=\mathbf{A}_{k} \mathbf{x}_{k}+\mathbf{B}_{k} \mathbf{u}_{k}+\mathbf{c}_{k}+\mathbf{v}_{k} \quad k=0,1, \ldots, N-1
$$

where $\mathbf{x}_{k}$ is the $n$-dimensional vector of states, $\mathbf{u}_{k}$ the $m$-dimensional vector of controls, the $\mathbf{W}_{k}, \boldsymbol{\Lambda}_{k}, \mathbf{w}_{k}$ and $\boldsymbol{\lambda}_{k}$ are penalty weights, $\mathbf{A}_{k}, \mathbf{B}_{k}$ and $\mathbf{c}_{k}$ are arrays of parameters of appropriate dimension and $\mathbf{v}_{k}$ an additive noise term. ${ }^{3}$

The expectation in (1) is taken over $\mathbf{v}_{k}, \mathbf{A}_{k}, \mathbf{B}_{k}$ and $\mathbf{c}_{k}$. It is assumed that these parameters follow a first-order Markov process of the form

$$
\boldsymbol{\theta}_{k+1}=\mathbf{D} \boldsymbol{\theta}_{k}+\boldsymbol{\eta}_{k}
$$

where $\mathbf{D}$ is a known matrix, $\boldsymbol{\eta}_{k}$ is a random vector and ${ }^{4}$

\footnotetext{
${ }^{3}$ As discussed in Kendrick (1981, 2002, Chapter 2), the $\mathbf{W}_{k}$ and $\boldsymbol{\Lambda}_{k}$ may be interpreted as penalty matrices on the deviations of the states and controls, respectively, from their desired paths and the $\mathbf{w}_{k}$ and $\boldsymbol{\lambda}_{k}$ as some known functions of the desired paths of the state and controls, respectively. The $\mathbf{w}_{k}$ and $\boldsymbol{\lambda}_{k}$ are zero when the desired paths of the state and controls, respectively, are 0 . In the engineering literature it is usually assumed that the $\mathbf{W}_{k}$ are positive semi-definite symmetric matrices and the $\boldsymbol{\Lambda}_{k}$ are positive definite symmetric matrices. See Bertsekas (2005, Chapter 4).

${ }^{4}$ It should be noticed that in Kendrick $(1981,2002)$ only the unknown parameters, either time-varying or constant, are included in $\boldsymbol{\theta}_{k}$. To go from the $\boldsymbol{\theta}_{k}$ as defined in the paper to that used in Kendrick, say $\boldsymbol{\theta}_{k}^{K}$, it suffices to pre-multiply $\boldsymbol{\theta}_{k}$ by the matrix $\mathbf{T}$ of dimension $r \times s$ where $r$ is the number of unknown parameters and $s$ is as in the text. Each row in $\mathbf{T}$ has 1 in the position associated with a certain unknown parameter and zero elsewhere. As an example consider a situation where $s=5$ but only the second and fourth parameter of vector $\boldsymbol{\theta}_{k}$ are unknown. Then the matrix $\mathbf{T}$ is $2 \times 5$ with 1 's in position $(1,2)$ and $(2,4)$ and 0 elsewhere.
} 


$$
\boldsymbol{\theta}_{k}=\left[\begin{array}{c}
\operatorname{vec}\left(\mathbf{A}_{k}\right) \\
\operatorname{vec}\left(\mathbf{B}_{k}\right) \\
\operatorname{vec}\left(\mathbf{c}_{k}\right)
\end{array}\right]
$$

is of dimension $(s \times 1)$, with $s=n \times n+n \times m+n$.

This formulation is general enough to model both time-varying and constant parameters. When a certain parameter, say the $i$-th parameter in $\boldsymbol{\theta}$, is assumed constant the corresponding row in $\mathbf{D}$ has 1 in the $i$-th column and zero elsewhere and the corresponding element in $\boldsymbol{\eta}_{k}$ is zero with zero variance. A parameter following a random walk has 1 in the appropriate position of the transition matrix and a positive variance.. Alternatively, if it is assumed to follow a return to normality model around a constant mean equation (5) describes the way its deviations change over time and the associated element in $\mathbf{D}$ is constrained to guarantee stationarity.

The noise vectors $\mathbf{v}_{k}$ and $\boldsymbol{\eta}_{k}$ are assumed independently distributed with ${ }^{5}$

$$
\begin{aligned}
\mathbf{v}_{k} & \sim N(\mathbf{0}, \mathbf{Q}) \\
\boldsymbol{\eta}_{k} & \sim N(\mathbf{0}, \mathbf{\Gamma})
\end{aligned}
$$

Furthermore, they are independent of the initial condition $\mathbf{x}_{\mathbf{0}}$, assumed given, and ${ }^{6}$

$$
\boldsymbol{\theta}_{0} \sim N\left(\boldsymbol{\theta}_{0 \mid 0}, \boldsymbol{\Sigma}_{0 \mid 0}^{\boldsymbol{\theta}}\right)
$$

The $s \times s$ covariance matrix looks like

$$
\boldsymbol{\Sigma}_{0 \mid 0}^{\boldsymbol{\theta} \boldsymbol{\theta}}=\left[\begin{array}{lll}
\boldsymbol{\Sigma}_{0 \mid 0}^{\mathrm{AA}} & \boldsymbol{\Sigma}_{0 \mid 0}^{\mathrm{AB}} & \boldsymbol{\Sigma}_{0 \mid 0}^{\mathrm{Ac}} \\
\bullet & \boldsymbol{\Sigma}_{0 \mid 0}^{\mathrm{BB}} & \boldsymbol{\Sigma}_{0 \mid 0}^{\mathrm{Bc}} \\
\bullet & \bullet & \Sigma_{0 \mid 0}^{\mathrm{cc}}
\end{array}\right]
$$

with $^{7}$

\footnotetext{
${ }^{5}$ When some of the parameters in $\boldsymbol{\theta}_{k}$ are assumed known the corresponding elements in $\boldsymbol{\eta}_{k}$ are zero and the associated variances and covariances are zero. Therefore, in general, the matrix $\boldsymbol{\Gamma}$ is symmetric and positive semi-definite in (7).

${ }^{6}$ When a certain parameter is constant and known the relative row and column in $\boldsymbol{\Sigma}_{0 \mid 0}^{\boldsymbol{\theta} \boldsymbol{\theta}}$ have zeroes. When it is constant but unknown the same row and column contain the covariances of its estimate at time zero included in $\boldsymbol{\theta}_{0 \mid 0}$. Some authors, for instance Harvey (1981, pages 104-106), prefer to use the notation $\left(\boldsymbol{\theta}_{0}-\boldsymbol{\theta}_{0 \mid 0}\right) \sim N\left(0, \boldsymbol{\Sigma}_{0 \mid 0}^{\boldsymbol{\theta} \boldsymbol{\theta}}\right)$, in place of (8), to indicate the distribution of a vector containing both fixed and random parameters. In the presence of measurement error $\mathbf{x}_{0}$ is usually assumed normally distributed with mean $\mathbf{x}_{0 \mid 0}$ and covariance $\boldsymbol{\Sigma}_{0 \mid 0}^{\mathbf{x}}$. See, e.g., Kendrick (1981, 2002, Chapter 10).

${ }^{7}$ Equation $(8)$ can be put in Kendrick's $(1981,2002)$ notation and using the $\mathbf{T}$ matrix defined in Footnote (4), that is $\boldsymbol{\theta}_{k}^{K} \equiv \mathbf{T} \boldsymbol{\theta}_{k} \sim N\left(\mathbf{T} \boldsymbol{\theta}_{k}, \mathbf{T} \boldsymbol{\Sigma}_{0 \mid 0}^{\boldsymbol{\theta} \boldsymbol{\theta}} \mathbf{T}^{\prime}\right)$. In the example discussed in that footnote the vector $\boldsymbol{\theta}_{k}^{K}$ is defined as
} 


$$
\begin{aligned}
& \boldsymbol{\Sigma}_{0 \mid 0}^{\mathbf{A}}=\text { the }\left(n^{2} \times n^{2}\right) \text { covariance matrix of the parameters in } \mathbf{A}_{0} ; \\
& \boldsymbol{\Sigma}_{0 \mid 0}^{\mathbf{A B}}=\text { the }\left(n^{2} \times n m\right) \text { matrix of covariances between the parameters in } \\
& \mathbf{A}_{0} \text { and } \mathbf{B}_{0} \text {; } \\
& \boldsymbol{\Sigma}_{0 \mid 0}^{\mathbf{A} \mathbf{c}}=\text { the }\left(n^{2} \times n\right) \text { matrix of covariances between the parameters in } \\
& \mathbf{A}_{0} \text { and } \mathbf{c}_{0} \text {; } \\
& \boldsymbol{\Sigma}_{0 \mid 0}^{\mathrm{BB}}=\text { the }(n m \times n m) \text { covariance matrix of the parameters in } \mathbf{B}_{0} \text {; } \\
& \boldsymbol{\Sigma}_{0 \mid 0}^{\mathbf{B c}}=\text { the }(n m \times n) \text { matrix of covariances between the parameters in } \\
& \mathbf{B}_{0} \text { and } \mathbf{c}_{0} \text {; } \\
& \boldsymbol{\Sigma}_{0 \mid 0}^{\mathrm{cc}}=\text { the }(n \times n) \text { covariance matrix of the parameters in } \mathbf{c}_{0}
\end{aligned}
$$

When the system parameters are jointly estimated this formulation guarantees that all the available information about the states, controls and parameters estimates and covariances is used in determining the optimal policy. If attention is focused only on a subsets of these parameters, for instance those included in the matrix $\mathbf{B}$ because they are "central to the trade-off between current control and estimation" (Beck and Wieland, 2002), and the others are treated as known then the computed control will be close to the optimal one only when the relevant parameter covariance matrices are zero. ${ }^{8}$

In the following pages this problem is solved by using dynamic programming methods and working backward in time following the procedures used in Kendrick (1981, 2002, Chapter 6) but with time varying parameters. Given $k=0$, first the problem is solved for period $N$ and then for period $N-1$. Having found the optimal policy at time $N-1$ whatever the state will be in that period we proceed backward. This leads to the solution for a generic period $j$ in the planning horizon. Then the optimal control for period zero is determined and the system is moved forward.

$$
\mathbf{T} \boldsymbol{\theta}_{k}=\left[\begin{array}{ccccc}
0 & 1 & 0 & 0 & 0 \\
0 & 0 & 0 & 1 & 0
\end{array}\right]\left[\begin{array}{c}
\theta_{1} \\
\theta_{2} \\
\theta_{3} \\
\theta_{4} \\
\theta_{5}
\end{array}\right]
$$

and it should be noticed that $\boldsymbol{\theta}_{k} \equiv \mathbf{T}^{\prime} \boldsymbol{\theta}_{k}^{K}$. Therefore the same matrix can be used to go from the notation of this paper to Kendrick's (1981, 2002) notation and in the opposite direction. This is extremely convenient from a computational point of view.

${ }^{8}$ The situation is similar to that occurring in statistics when the joint confidence region of two estimated parameters is constructed starting from the individual confidence intervals instead of the associated F-statistic. The rectangular region derived with the former approach will be close to the ellipse obtained from the latter one only when the covariance between the two parameters is zero. See, e.g., Johnston (1984, figure 5.1). 


\section{Period $N$}

Using the notation in Kendrick (1981, 2002, Chapter 6) the optimal expected cost to go at period 0 , with $N$ periods remaining, is written as

$$
J_{N}^{*}=\min _{\mathbf{u}_{0}} E\left\{\ldots \min _{\mathbf{u}_{N-2}} E\left\{\min _{\mathbf{u}_{N-1}} E\left\{C_{N} \mid \mathcal{P}^{N-1}\right\} \mid \mathcal{P}^{N-2}\right\} \cdots \mid \mathcal{P}^{0}\right\}
$$

where $\mathcal{P}^{j}$, for $j=0, \ldots, N-1$, is defined as the means and covariances of the unknown parameters at time $j$. Alternatively equation (9) can be written as $^{9}$

$$
J_{N}^{*}=\min _{\mathbf{u}_{0}} E_{0}\left\{\cdots \min _{\mathbf{u}_{N-2}} E_{N-2}\left\{\min _{\mathbf{u}_{N-1}} E_{N-1}\left\{C_{N}\right\}\right\} \cdots\right\}
$$

where the subscript on the expectation operator indicates that the expectation on the parameters is conditional on the information on the parameters available at that time, that is

$$
E_{N-1}\left\{C_{N}\right\} \equiv E\left\{C_{N} \mid \mathcal{P}^{N-1}\right\}
$$

From the nested expression (10) it follows that each control $\mathbf{u}_{j}$ must be chosen with the information on the parameters available at time $j$.

The typical situation when $\mathcal{P}^{j}$, for $j=0, \ldots, N-1$, is known at time 0 , is when the parameters are identically and independently distributed. ${ }^{10}$ Then the exact EOF, or cautionary myopic rule in Beck and Wieland (2002) terminology, can be found. When the parameters are modeled as in equations (5)-(6) this is clearly not true and an approximation to the dynamic programming algorithm is needed. Following Chow (1973, 1975a, Chapter 10), the approximation presented in these pages uses all the information available at time zero, namely the distribution associated with $\boldsymbol{\theta}_{0}$, and replaces the expectations on the parameters conditional on the knowledge available at the time the control is applied, say time $j$ in the planning horizon, in equation (10) with the corresponding conditional expectations at time zero. As stated in the Introduction, this means to ignore possible revisions of the density (of the parameters) by observations on the (states and controls) from

\footnotetext{
${ }^{9}$ In general at time $j$, with $N-j$ periods remaining, the summation in equation (1) goes from $k=j$ to $N-1$ and the associated cost is denoted by $C_{N-j}$. Then equation (10) looks like

$$
J_{N-j}^{*}=\min _{\mathbf{u}_{j}} E_{j}\left\{\cdots \min _{\mathbf{u}_{N-2}} E_{N-2}\left\{\min _{\mathbf{u}_{N-1}} E_{N-1}\left\{C_{N-j}\right\}\right\} \cdots\right\}
$$

${ }^{10}$ This is the case considered in Kendrick (1981, 2002, Chapter 6) and usually discussed in the engineering literature. See Bertsekas (2005, Chapter 4).
} 
.... period 1 on (Chow 1975a, page 223). ${ }^{11}$

As discussed in Kendrick (1981, Chapter 2) in dynamic programming problems, for any arbitrary time period $j$, the optimal cost-to-go with $N-j$ periods remaining will equal the minimum over the choice of the control at time $j$ of the cost incurred during period $j$ plus the optimal cost-to-go with $N-(j+1)$ periods remaining. Therefore the approximate optimal feedback rule for problem (1)-(8) is solved starting from the last period and working backward toward the initial period.

In period $N$ no control is chosen and from equation (2) it follows that the optimal cost is

$$
J_{0}^{*}=\frac{1}{2} \mathbf{x}_{N}^{\prime} \mathbf{W}_{N} \mathbf{x}_{N}+\mathbf{w}_{N}^{\prime} \mathbf{x}_{N}
$$

In general, see e.g. Kendrick (1981, 2002, Chapter 2), the optimal cost-to-go for the quadratic linear problem, sometimes called the regulatory problem, in a certain period is a quadratic function of the state of the system in that period. So the expected cost-to-go with zero periods to go may be written as

$$
J_{0}^{*}=\frac{1}{2} \mathbf{x}_{N}^{\prime} \mathbf{K}_{N} \mathbf{x}_{N}+\mathbf{p}_{N}^{\prime} \mathbf{x}_{N}+\nu_{N}
$$

where the scalar $\nu_{N}$, the vector $\mathbf{p}_{N}$, and the matrix $\mathbf{K}_{N}$ are the parameters of the quadratic function to be determined recursively in the optimization procedure. $^{12}$

Then comparing equation (11) with equation (12) one obtains the terminal conditions for the Riccati equations, namely

$$
\mathbf{K}_{N}=\mathbf{W}_{N}, \mathbf{p}_{N}=\mathbf{w}_{N} \text { and } \nu_{N}=0
$$

\section{Period $N-1$}

The optimal cost-to-go in period $N-1$ can be written as

$$
J_{1}^{*}=\min _{\mathbf{u}_{N-1}} E_{N-1}\left\{J_{0}^{*}+L_{N-1}\left(\mathbf{x}_{N-1}, \mathbf{u}_{N-1}\right)\right\}
$$

\footnotetext{
${ }^{11}$ Chow $(1973,1975 a)$ uses a similar approximation when dealing with unknown constant parameters. By the law of iterative predictions it follows, that the suggested linear approximation is based on the minimum mean-square-error estimators of the future unknown values of the stochastic parameters when disturbances are assumed gaussian. See, e.g., Hamilton (1994, Section 4.5) and Anderson and Moore (1979, Theorem 3.1, page 26).

${ }^{12}$ The term $\nu$ is sometimes omitted because "it does not affect the optimal control path but only the optimal cost-to-go" (Kendrick (1981, page 48))
} 
where $J_{0}^{*}$ is the optimal cost-to-go with 0 periods remaining and $L_{N-1}$ is the cost incurred in period $N-1$. Substituting equation (3) and equation (12), with $\nu_{N}=0$, into equation (14) yields

$$
\begin{gathered}
J_{1}^{*}=\min _{\mathbf{u}_{N-1}} E_{N-1}\left\{\frac{1}{2} \mathbf{x}_{N}^{\prime} \mathbf{K}_{N} \mathbf{x}_{N}+\mathbf{p}_{N}^{\prime} \mathbf{x}_{N}+\frac{1}{2} \mathbf{x}_{N-1}^{\prime} \mathbf{W}_{N-1} \mathbf{x}_{N-1}+\mathbf{w}_{N-1}^{\prime} \mathbf{x}_{N-1}\right. \\
\left.+\mathbf{x}_{N-1}^{\prime} \mathbf{F}_{N-1} \mathbf{u}_{N-1}+\frac{1}{2} \mathbf{u}_{N-1}^{\prime} \boldsymbol{\Lambda}_{N-1} \mathbf{u}_{N-1}+\boldsymbol{\lambda}_{N-1}^{\prime} \mathbf{u}_{N-1}\right\}
\end{gathered}
$$

This expression gives the optimal cost-to-go in terms of $\left(\mathbf{x}_{N}, \mathbf{x}_{N-1}, \mathbf{u}_{N-1}\right)$. After replacing $\mathbf{x}_{N}$ with the system equations given in equation (4), equation (15) looks like

$$
\begin{aligned}
J_{1}^{*}= & \min _{\mathbf{u}_{N-1}} E_{N-1}\left\{\frac{1}{2}\left(\mathbf{A}_{N-1} \mathbf{x}_{N-1}+\mathbf{B}_{N-1} \mathbf{u}_{N-1}+\mathbf{c}_{N-1}+\mathbf{v}_{N-1}\right)^{\prime} \mathbf{K}_{N}\right. \\
& \times\left(\mathbf{A}_{N-1} \mathbf{x}_{N-1}+\mathbf{B}_{N-1} \mathbf{u}_{N-1}+\mathbf{c}_{N-1}+\mathbf{v}_{N-1}\right) \\
& +\mathbf{p}_{N}^{\prime}\left(\mathbf{A}_{N-1} \mathbf{x}_{N-1}+\mathbf{B}_{N-1} \mathbf{u}_{N-1}+\mathbf{c}_{N-1}+\mathbf{v}_{N-1}\right)+ \\
& \frac{1}{2} \mathbf{x}_{N-1}^{\prime} \mathbf{W}_{N-1} \mathbf{x}_{N-1}+\mathbf{w}_{N-1}^{\prime} \mathbf{x}_{N-1}+\mathbf{x}_{N-1}^{\prime} \mathbf{F}_{N-1} \mathbf{u}_{N-1}+ \\
& \left.\frac{1}{2} \mathbf{u}_{N-1}^{\prime} \boldsymbol{\Lambda}_{N-1} \mathbf{u}_{N-1}+\boldsymbol{\lambda}_{N-1}^{\prime} \mathbf{u}_{N-1}\right\}
\end{aligned}
$$

which depends only on $\mathbf{x}_{N-1}$ and $\mathbf{u}_{N-1}$. Multiplying the various terms in (16) and taking the parameter expectations conditional on the information available at time $N-1$, yields ${ }^{13}$

\footnotetext{
${ }^{13}$ See, e.g., Kendrick (1981, page 46$)$.
} 


$$
\begin{aligned}
J_{1}^{*}= & \min _{u_{N-1}}\left\{\frac { 1 } { 2 } \left[\mathbf{x}_{N-1}^{\prime} E_{N-1}\left(\mathbf{A}_{N-1}^{\prime} \mathbf{K}_{N} \mathbf{A}_{N-1}\right) \mathbf{x}_{N-1}\right.\right. \\
& +\mathbf{x}_{N-1}^{\prime} E_{N-1}\left(\mathbf{A}_{N-1}^{\prime} \mathbf{K}_{N} \mathbf{B}_{N-1}\right) \mathbf{u}_{N-1} \\
& +\mathbf{x}_{N-1}^{\prime} E_{N-1}\left(\mathbf{A}_{N-1}^{\prime} \mathbf{K}_{N} \mathbf{c}_{N-1}\right) \\
& +\mathbf{u}_{N-1}^{\prime} E_{N-1}\left(\mathbf{B}_{N-1}^{\prime} \mathbf{K}_{N} \mathbf{A}_{N-1}\right) \mathbf{x}_{N-1} \\
& +\mathbf{u}_{N-1}^{\prime} E_{N-1}\left(\mathbf{B}_{N-1}^{\prime} \mathbf{K}_{N} \mathbf{B}_{N-1}\right) \mathbf{u}_{N-1} \\
& +\mathbf{u}_{N-1}^{\prime} E_{N-1}\left(\mathbf{B}_{N-1}^{\prime} \mathbf{K}_{N} \mathbf{c}_{N-1}\right) \\
& +E_{N-1}\left(\mathbf{c}_{N-1}^{\prime} \mathbf{K}_{N} \mathbf{A}_{N-1}\right) \mathbf{x}_{N-1}+E_{N-1}\left(\mathbf{c}_{N-1}^{\prime} \mathbf{K}_{N} \mathbf{B}_{N-1}\right) \mathbf{u}_{N-1} \\
& \left.+E_{N-1}\left(\mathbf{c}_{N-1}^{\prime} \mathbf{K}_{N} \mathbf{c}_{N-1}\right)+E_{N-1}\left(\mathbf{v}_{N-1}^{\prime} \mathbf{K}_{N} \mathbf{v}_{N-1}\right)\right] \\
& +\mathbf{p}_{N}^{\prime} E_{N-1}\left(\mathbf{A}_{N-1}\right) \mathbf{x}_{N-1}+\mathbf{p}_{N}^{\prime} E_{N-1}\left(\mathbf{B}_{N-1}\right) \mathbf{u}_{N-1} \\
& +\mathbf{p}_{N}^{\prime} E_{N-1}\left(\mathbf{c}_{N-1}\right) \\
& +\frac{1}{2} \mathbf{x}_{N-1}^{\prime} \mathbf{W}_{N-1} \mathbf{x}_{N-1}+\mathbf{w}_{N-1}^{\prime} \mathbf{x}_{N-1}+\mathbf{x}_{N-1}^{\prime} \mathbf{F}_{N-1} \mathbf{u}_{N-1} \\
& \left.+\frac{1}{2} \mathbf{u}_{N-1}^{\prime} \mathbf{\Lambda}_{N-1} \mathbf{u}_{N-1}+\boldsymbol{\lambda}_{N-1}^{\prime} \mathbf{u}_{N-1}\right\}
\end{aligned}
$$

with the expectations involving only $\mathbf{v}_{N-1}$ and the covariances between $\mathbf{v}_{N-1}$ and the time-varying parameters omitted because they are 0 by assumption.

Minimizing equation (17) with respect to the vector of controls yields the first order condition, namely

$$
\begin{aligned}
& E_{N-1}\left(\mathbf{B}_{N-1}^{\prime} \mathbf{K}_{N} \mathbf{A}_{N-1}\right) \mathbf{x}_{N-1}+E_{N-1}\left(\mathbf{B}^{\prime}{ }_{N-1} \mathbf{K}_{N} \mathbf{B}_{N-1}\right) \mathbf{u}_{N-1}^{*} \\
& \quad+E_{N-1}\left(\mathbf{B}^{\prime}{ }_{N-1} \mathbf{K}_{N} \mathbf{c}_{N-1}\right)+E_{N-1}\left(\mathbf{B}^{\prime}{ }_{N-1}\right) \mathbf{p}_{N}+ \\
& \quad \mathbf{F}_{N-1}^{\prime} \mathbf{x}_{N-1}+\boldsymbol{\Lambda}_{N-1} \mathbf{u}_{N-1}^{*}+\boldsymbol{\lambda}_{N-1}=0,
\end{aligned}
$$

which implies that the cost minimizing control, or feedback rule, for time $N-1$ is

$$
\mathbf{u}_{N-1}^{*}=\mathbf{G}_{N-1} \mathbf{x}_{N-1}+\mathbf{g}_{N-1}
$$

where

$$
\begin{aligned}
\mathbf{G}_{N-1}= & -\left[\boldsymbol{\Lambda}_{N-1}+E_{N-1}\left(\mathbf{B}_{N-1}^{\prime} \mathbf{K}_{N} \mathbf{B}_{N-1}\right)\right]^{-1} \\
& \times\left[E_{N-1}\left(\mathbf{B}_{N-1}^{\prime} \mathbf{K}_{N} \mathbf{A}_{N-1}\right)+\mathbf{F}_{N-1}^{\prime}\right] \\
\mathbf{g}_{N-1}= & -\left[\boldsymbol{\Lambda}_{N-1}+E_{N-1}\left(\mathbf{B}_{N-1}^{\prime} \mathbf{K}_{N} \mathbf{B}_{N-1}\right)\right]^{-1} \\
& \times\left[E_{N-1}\left(\mathbf{B}_{N-1}^{\prime} \mathbf{K}_{N} \mathbf{c}_{N-1}\right)+E_{N-1}\left(\mathbf{B}_{N-1}^{\prime}\right) \mathbf{p}_{N}+\boldsymbol{\lambda}_{N-1}\right]
\end{aligned}
$$


which resembles the standard stochastic case, that is the case where the para-meter matrices are assumed either identically and independently distributed or unknown but constant, except for the fact that here the parameter expectations are conditional on the information available at time $N-1$ . The feedback rule (19), (20) and (21) provide the optimality condition sought for period $N-1$. However the optimal feedback control equation (19) is not a linear function of $x_{N-1}$ because the feedback arrays in (20) and (21) depend on conditional expectations that are clearly functions of the observations on the states and controls from period 1 on. Equation (19) can be treated as a linear function of current states when the needed expectations are approximated by the corresponding conditional expectations at time 0 . The approximated optimal expected cost-to-g, denoted by $\hat{J}_{1}^{*}$ from now on to stress the fact that it is different from $J_{1}^{*}$, is obtained replacing the approximated feedback rule in the cost functional, e.g.

$$
\begin{aligned}
\hat{J}_{1}^{*}= & \left\{\frac { 1 } { 2 } \left[\mathbf{x}_{N-1}^{\prime} E_{0}\left(\mathbf{A}_{N-1}^{\prime} \mathbf{K}_{N} \mathbf{A}_{N-1}\right) \mathbf{x}_{N-1}\right.\right. \\
& +\mathbf{x}_{N-1}^{\prime} E_{0}\left(\mathbf{A}_{N-1}^{\prime} \mathbf{K}_{N} \mathbf{B}_{N-1}\right)\left(\mathbf{G}_{N-1} \mathbf{x}_{N-1}+\mathbf{g}_{N-1}\right) \\
& +\mathbf{x}_{N-1}^{\prime} E_{0}\left(\mathbf{A}_{N-1}^{\prime} \mathbf{K}_{N} \mathbf{c}_{N-1}\right) \\
& +\left(\mathbf{G}_{N-1} \mathbf{x}_{N-1}+\mathbf{g}_{N-1}\right)^{\prime} E_{0}\left(\mathbf{B}_{N-1}^{\prime} \mathbf{K}_{N} \mathbf{A}_{N-1}\right) \mathbf{x}_{N-1} \\
& +\left(\mathbf{G}_{N-1} \mathbf{x}_{N-1}+\mathbf{g}_{N-1}\right)^{\prime} E_{0}\left(\mathbf{B}_{N-1}^{\prime} \mathbf{K}_{N} \mathbf{B}_{N-1}\right)\left(\mathbf{G}_{N-1} \mathbf{x}_{N-1}+\mathbf{g}_{N-1}\right) \\
& +\left(\mathbf{G}_{N-1} \mathbf{x}_{N-1}+\mathbf{g}_{N-1}\right)^{\prime} E_{0}\left(\mathbf{B}_{N-1}^{\prime} \mathbf{K}_{N} \mathbf{c}_{N-1}\right) \\
& +E_{0}\left(\mathbf{c}_{N-1}^{\prime} \mathbf{K}_{N} \mathbf{A}_{N-1}\right) \mathbf{x}_{N-1}+E_{0}\left(\mathbf{c}_{N-1}^{\prime} \mathbf{K}_{N} \mathbf{B}_{N-1}\right)\left(\mathbf{G}_{N-1} \mathbf{x}_{N-1}+\mathbf{g}_{N-1}\right) \\
& \left.+E_{0}\left(\mathbf{c}_{N-1}^{\prime} \mathbf{K}_{N} \mathbf{c}_{N-1}\right)+E_{0}\left(\mathbf{v}_{N-1}^{\prime} \mathbf{K}_{N} \mathbf{v}_{N-1}\right)\right] \\
& +\mathbf{p}_{N}^{\prime} E_{0}\left(\mathbf{A}_{N-1}\right) \mathbf{x}_{N-1}+\mathbf{p}_{N}^{\prime} E_{0}\left(\mathbf{B}_{N-1}\right)\left(\mathbf{G}_{N-1} \mathbf{x}_{N-1}+\mathbf{g}_{N-1}\right) \\
& +\mathbf{p}_{N}^{\prime} E_{0}\left(\mathbf{c}_{N-1}\right)+\frac{1}{2} \mathbf{x}_{N-1}^{\prime} \mathbf{W}_{N-1} \mathbf{x}_{N-1}+\mathbf{w}_{N-1}^{\prime} \mathbf{x}_{N-1} \\
& +\mathbf{x}_{N-1}^{\prime} \mathbf{F}_{N-1}\left(\mathbf{G}_{N-1} \mathbf{x}_{N-1}+\mathbf{g}_{N-1}\right) \\
& +\frac{1}{2}\left(\mathbf{G}_{N-1} \mathbf{x}_{N-1}+\mathbf{g}_{N-1}\right)^{\prime} \mathbf{\Lambda}_{N-1}\left(\mathbf{G}_{N-1} \mathbf{x}_{N-1}+\mathbf{g}_{N-1}\right) \\
& \left.+\boldsymbol{\lambda}_{N-1}^{\prime}\left(\mathbf{G}_{N-1} \mathbf{x}_{N-1}+\mathbf{g}_{N-1}\right)\right\}
\end{aligned}
$$

At this point, using equation (13) and simplifying and rearranging the terms gives 


$$
\begin{aligned}
\hat{J}_{1}^{*}= & \frac{1}{2} \mathbf{x}_{N-1}^{\prime}\left\{\mathbf{W}_{N-1}+E_{0}\left(\mathbf{A}_{N-1}^{\prime} \mathbf{W}_{N} \mathbf{A}_{N-1}\right)\right. \\
& 2\left[E_{0}\left(\mathbf{A}_{N-1}^{\prime} \mathbf{W}_{N} \mathbf{B}_{N-1}\right)+\mathbf{F}_{N-1}\right] \mathbf{G}_{N-1} \\
& \left.+\mathbf{G}_{N-1}^{\prime}\left[E_{0}\left(\mathbf{B}_{N-1}^{\prime} \mathbf{W}_{N} \mathbf{B}_{N-1}\right)+\mathbf{\Lambda}_{N-1}\right] \mathbf{G}_{N-1}\right\} \mathbf{x}_{N-1} \\
& +\mathbf{x}_{N-1}^{\prime}\left\{\left[E_{0}\left(\mathbf{A}_{N-1}^{\prime} \mathbf{W}_{N} \mathbf{B}_{N-1}\right)+\mathbf{F}_{N-1}\right] \mathbf{g}_{N-1}\right. \\
& +\mathbf{G}_{N-1}^{\prime}\left[E_{0}\left(\mathbf{B}_{N-1}^{\prime} \mathbf{W}_{N} \mathbf{B}_{N-1}\right)+\boldsymbol{\Lambda}_{N-1}\right] \mathbf{g}_{N-1} \\
& +E_{0}\left(\mathbf{A}_{N-1}^{\prime} \mathbf{W}_{N} \mathbf{c}_{N-1}\right)+E_{0}\left(\mathbf{A}_{N-1}^{\prime}\right) \mathbf{w}_{N} \\
& \left.+\mathbf{G}_{N-1}^{\prime}\left[E_{0}\left(\mathbf{B}_{N-1}^{\prime} \mathbf{W}_{N} \mathbf{c}_{N-1}\right)+E_{0}\left(\mathbf{B}_{N-1}^{\prime}\right) \mathbf{w}_{N}+\boldsymbol{\lambda}_{N-1}\right]+\mathbf{w}_{N-1}\right\} \\
& +\frac{1}{2}\left\{\mathbf{g}_{N-1}^{\prime}\left[E_{0}\left(\mathbf{B}_{N-1}^{\prime} \mathbf{W}_{N} \mathbf{B}_{N-1}\right)+\mathbf{\Lambda}_{N-1}\right] \mathbf{g}_{N-1}\right. \\
& +2 \mathbf{g}_{N-1}^{\prime}\left[E_{0}\left(\mathbf{B}_{N-1}^{\prime} \mathbf{W}_{N} \mathbf{c}_{N-1}\right)+E_{0}\left(\mathbf{B}_{N-1}^{\prime}\right) \mathbf{w}_{N}+\boldsymbol{\lambda}_{N-1}\right] \\
& \left.+2 \mathbf{w}_{N}^{\prime} E_{0}\left(\mathbf{c}_{N-1}\right)+E_{0}\left(\mathbf{c}_{N-1}^{\prime} \mathbf{W}_{N} \mathbf{c}_{N-1}\right)+E_{0}\left(\mathbf{v}_{N-1}^{\prime} \mathbf{W}_{N} \mathbf{v}_{N-1}\right)\right\}
\end{aligned}
$$

But using the approximations equations (20) and (21) it follows that

$$
\begin{aligned}
& \mathbf{G}_{N-1}^{\prime}\left[E_{0}\left(\mathbf{B}_{N-1}^{\prime} \mathbf{W}_{N} \mathbf{B}_{N-1}\right)+\boldsymbol{\Lambda}_{N-1}\right] \mathbf{G}_{N-1}= \\
& -\left[E_{0}\left(\mathbf{B}_{N-1}^{\prime} \mathbf{K}_{N} \mathbf{A}_{N-1}\right)+\mathbf{F}_{N-1}^{\prime}\right]^{\prime} \mathbf{G}_{N-1} \\
& \mathbf{G}^{\prime}{ }_{N-1}\left[E_{0}\left(\mathbf{B}_{N-1}^{\prime} \mathbf{W}_{N} \mathbf{B}_{N-1}\right)+\boldsymbol{\Lambda}_{N-1}\right] \mathbf{g}_{N-1}= \\
& -\left[E_{0}\left(\mathbf{B}_{N-1}^{\prime} \mathbf{K}_{N} \mathbf{A}_{N-1}\right)+\mathbf{F}_{N-1}^{\prime}\right]^{\prime} \mathbf{g}_{N-1} \\
& \mathbf{G}^{\prime}{ }_{N-1}\left[E_{0}\left(\mathbf{B}_{N-1}^{\prime} \mathbf{W}_{N} \mathbf{c}_{N-1}\right)+E_{0}\left(\mathbf{B}_{N-1}^{\prime}\right) \mathbf{w}_{N}+\boldsymbol{\lambda}_{N-1}\right]= \\
& {\left[E_{0}\left(\mathbf{B}^{\prime}{ }_{N-1} \mathbf{K}_{N} \mathbf{A}_{N-1}\right)+\mathbf{F}_{N-1}^{\prime}\right]^{\prime} \mathbf{g}_{N-1}} \\
& \mathbf{g}_{N-1}^{\prime}\left[E_{0}\left(\mathbf{B}_{N-1}^{\prime} \mathbf{W}_{N} \mathbf{B}_{N-1}\right)+\boldsymbol{\Lambda}_{N-1}\right] \mathbf{g}_{N-1}= \\
& -\mathbf{g}_{N-1}^{\prime}\left[E_{0}\left(\mathbf{B}_{N-1}^{\prime} \mathbf{W}_{N} \mathbf{c}_{N-1}\right)+E_{0}\left(\mathbf{B}_{N-1}^{\prime}\right) \mathbf{w}_{N}+\boldsymbol{\lambda}_{N-1}\right]
\end{aligned}
$$

Therefore equation (23) can be rewritten as

$$
\hat{J}_{1}^{*}=\frac{1}{2} \mathbf{x}_{N-1}^{\prime} \mathbf{K}_{N-1} \mathbf{x}_{N-1}+\mathbf{x}_{N-1}^{\prime} \mathbf{p}_{N-1}+\nu_{N-1}
$$

with 


$$
\begin{aligned}
\mathbf{K}_{N-1} & =\mathbf{W}_{N-1}+E_{0}\left(\mathbf{A}_{N-1}^{\prime} \mathbf{K}_{N} \mathbf{A}_{N-1}\right) \\
& -\left[E_{0}\left(\mathbf{A}_{N-1}^{\prime} \mathbf{K}_{N} \mathbf{B}_{N-1}\right)+\mathbf{F}_{N-1}\right]\left[\mathbf{\Lambda}_{N-1}+E_{0}\left(\mathbf{B}_{N-1}^{\prime} \mathbf{K}_{N} \mathbf{B}_{N-1}\right)\right]^{-1} \\
& \times\left[E_{0}\left(\mathbf{B}_{N-1}^{\prime} \mathbf{K}_{N} \mathbf{A}_{N-1}\right)+\mathbf{F}_{N-1}^{\prime}\right] \\
\mathbf{p}_{N-1} & =E_{0}\left(\mathbf{A}_{N-1}^{\prime} \mathbf{K}_{N} \mathbf{c}_{N-1}\right)+E_{0}\left(\mathbf{A}_{N-1}^{\prime}\right) \mathbf{p}_{N}+\mathbf{w}_{N-1} \\
& -\left[E_{0}\left(\mathbf{A}_{N-1}^{\prime} \mathbf{K}_{N} \mathbf{B}_{N-1}\right)+\mathbf{F}_{N-1}\right]\left[\boldsymbol{\Lambda}_{N-1}+E_{0}\left(\mathbf{B}_{N-1}^{\prime} \mathbf{K}_{N} \mathbf{B}_{N-1}\right)\right]^{-1} \\
& \times\left[E_{0}\left(\mathbf{B}_{N-1}^{\prime} \mathbf{K}_{N} \mathbf{c}_{N-1}+E_{0}\left(\mathbf{B}_{N-1}^{\prime}\right) \mathbf{p}_{N}+\boldsymbol{\lambda}_{N-1}\right]\right. \\
\nu_{N-1} & =\frac{1}{2}\left\{-\left[E_{0}\left(\mathbf{B}_{N-1}^{\prime} \mathbf{K}_{N} \mathbf{c}_{N-1}\right)+E_{0}\left(\mathbf{B}_{N-1}^{\prime}\right) \mathbf{p}_{N}+\boldsymbol{\lambda}_{N-1}\right]^{\prime}\right. \\
& \times\left[\boldsymbol{\Lambda}_{N-1}+E_{0}\left(\mathbf{B}_{N-1}^{\prime} \mathbf{K}_{N} \mathbf{B}_{N-1}\right)\right]^{-1} \\
& \times\left[E_{0}\left(\mathbf{B}_{N-1}^{\prime} \mathbf{K}_{N} \mathbf{c}_{N-1}\right)+E_{0}\left(\mathbf{B}_{N-1}^{\prime}\right) \mathbf{w}_{N}+\boldsymbol{\lambda}_{N-1}\right] \\
& \left.+2 \mathbf{p}_{N}^{\prime} E_{0}\left(\mathbf{c}_{N-1}\right)+E_{0}\left(\mathbf{c}_{N-1}^{\prime} \mathbf{K}_{N} \mathbf{c}_{N-1}\right)+E_{0}\left(\mathbf{v}_{N-1}^{\prime} \mathbf{K}_{N} \mathbf{v}_{N-1}\right)\right\}
\end{aligned}
$$

Then, as noticed in Chow (1975a, page 231), the approximated minimum expected cost for period $N-1$ in equation (24) can be treated as a quadratic function of state in that period. The approximated Riccati equations (25), (26) and (27) look similar to those in the standard stochastic parameter case except for the parameter expectation being conditional on the information available at time $0{ }^{14}$ This process can be repeated backward for periods $N-1, N-2, \ldots$ and so on and so forth.

\section{$5 \quad$ Period $j$}

For a generic period $j$ in the planning horizon, from period 0 to $N-1$, the optimal cost-to-go in dynamic programming problems can be written as

$$
J_{N-j}^{*}=\min _{\mathbf{u}_{j}} E_{j}\left\{J_{N-(j+1)}^{*}+L_{j}\left(\mathbf{x}_{j}, \mathbf{u}_{j}\right)\right\}
$$

where $J_{N-(j+1)}^{*}$ is the optimal cost-to-go with $N-(j+1)$ periods remaining. Proceeding as in the case $j=N-1$ yields

$$
\mathbf{u}_{j}^{*}=\mathbf{G}_{j} \mathbf{x}_{j}+\mathbf{g}_{j}
$$

where

$$
\begin{aligned}
\mathbf{G}_{j}=- & {\left[\boldsymbol{\Lambda}_{j}+E_{j}\left(\mathbf{B}_{j}^{\prime} \mathbf{K}_{j+1} \mathbf{B}_{j}\right)\right]^{-1}\left[E_{j}\left(\mathbf{B}_{j}^{\prime} \mathbf{K}_{j+1} \mathbf{A}_{j}\right)+\mathbf{F}^{\prime}{ }_{j}\right] } \\
\mathbf{g}_{j}=- & {\left[\boldsymbol{\Lambda}_{j}+E_{j}\left(\mathbf{B}_{j}^{\prime} \mathbf{K}_{j+1} \mathbf{B}_{j}\right)\right]^{-1} } \\
& \times\left[E_{j}\left(\mathbf{B}^{\prime}{ }_{j} \mathbf{K}_{j+1} \mathbf{c}_{j}\right)+E_{j}\left(\mathbf{B}^{\prime}{ }_{j}\right) \mathbf{w}_{j+1}+\boldsymbol{\lambda}_{j}\right]
\end{aligned}
$$

\footnotetext{
${ }^{14}$ See, e.g., equation (6.29) in Kendrick (1981, 2002).
} 
As in the previous section the optimal feedback control equation (29) is not a linear function of $x_{j}$ because of the feedback arrays in (30) and (31). The control is a linear function of current states when the needed expectations are approximated by the corresponding conditional expectations at time 0 . Then the approximated optimal expected cost-to-go can be rewritten as

$$
\hat{J}_{j}^{*}=\frac{1}{2} \mathbf{x}_{j}^{\prime} \mathbf{K}_{j} \mathbf{x}_{j}+\mathbf{x}^{\prime}{ }_{j} \mathbf{p}_{j}+\nu_{j}
$$

with

$$
\begin{aligned}
\mathbf{K}_{j}= & \mathbf{W}_{j}+E_{0}\left(\mathbf{A}_{j}^{\prime} \mathbf{K}_{j+1} \mathbf{A}_{j}\right) \\
& -\left[E_{0}\left(\mathbf{A}_{j}^{\prime} \mathbf{K}_{j+1} \mathbf{B}_{j}\right)+\mathbf{F}_{j}\right]\left[\mathbf{\Lambda}_{j}+E_{0}\left(\mathbf{B}_{j}^{\prime} \mathbf{K}_{j+1} \mathbf{B}_{j}\right)\right]^{-1} \\
& \left.\times\left[E_{0} \mathbf{B}_{j}^{\prime} \mathbf{K}_{j+1} \mathbf{A}_{j}\right)+\mathbf{F}_{j}^{\prime}\right] \\
\mathbf{p}_{j}= & E_{0}\left(\mathbf{A}_{j}^{\prime} \mathbf{K}_{j+1} \mathbf{c}_{j}\right)+E_{0}\left(\mathbf{A}_{j}^{\prime}\right) \mathbf{p}_{j+1}+\mathbf{w}_{j}-\left[E_{0}\left(\mathbf{A}_{j}^{\prime} \mathbf{K}_{j+1} \mathbf{B}_{j}\right)+\mathbf{F}_{j}\right] \\
& \times\left[\boldsymbol{\Lambda}_{j}+E_{0}\left(\mathbf{B}_{j}^{\prime} \mathbf{K}_{j+1} \mathbf{B}_{j}\right)\right]^{-1}\left[E_{0}\left(\mathbf{B}_{j}^{\prime} \mathbf{K}_{j+1} \mathbf{c}_{j}\right)+E_{0}\left(\mathbf{B}_{j}^{\prime}\right) \mathbf{p}_{j+1}+\boldsymbol{\lambda}_{j}\right] \\
\nu_{j}= & \frac{1}{2}\left\{-\left[E_{0}\left(\mathbf{B}_{j}^{\prime} \mathbf{K}_{j+1} \mathbf{c}_{j}\right)+E_{0}\left(\mathbf{B}_{j}^{\prime}\right) \mathbf{p}_{j+1}+\boldsymbol{\lambda}_{j}\right]^{\prime}\right. \\
& \times\left[\boldsymbol{\Lambda}_{j}+E_{0}\left(\mathbf{B}_{j}^{\prime} \mathbf{K}_{j+1} \mathbf{B}_{j}\right)\right]^{-1}\left[E_{0}\left(\mathbf{B}_{j}^{\prime} \mathbf{K}_{j+1} \mathbf{c}_{j}\right)+E_{0}\left(\mathbf{B}_{j}^{\prime}\right) \mathbf{p}_{j+1}+\boldsymbol{\lambda}_{j}\right]+ \\
& \left.2 \mathbf{p}_{j+1}^{\prime} E_{0}\left(\mathbf{c}_{j}\right)+E_{0}\left(\mathbf{c}_{j}^{\prime} \mathbf{K}_{j+1} \mathbf{c}_{j}\right)+E_{0}\left(\mathbf{v}_{j}^{\prime} \mathbf{K}_{j+1} \mathbf{v}_{j}\right)\right\}
\end{aligned}
$$

As in the previous section, the only difference with respect to the standard stochastic case is that the parameter expectations in the approximated Riccati equations are conditional on the information available at the beginning of the planning horizon.

In summary, similarly to the random parameter case where parameters vary randomly around a constant mean considered in Kendrick (1981, page 4349 ), the problem at period zero is solved using the terminal conditions $\mathbf{K}_{N}$ $=\mathbf{W}_{N}, \mathbf{p}_{N}=\mathbf{w}_{N}$ and $\mathbf{v}_{N}=0$ in equations (33), (34) and (35) to integrate the Riccati equations backward in time. Then the approximated $\mathbf{G}$ and $\mathbf{g}$ elements can be computed for all time periods. Using the initial conditions for the state vector $\mathbf{x}_{0}$ and the parameters, the approximated feedback rule is applied to compute $\mathbf{u}_{0}$. As soon as the new observation on the state $\mathbf{x}_{1}$ becomes available the estimate of the parameter vector can be updated ${ }^{15}$ and the exercise is repeated for period $k=1$ with all the parameter expectations conditional on the information available at time 1 , then for period $k$ $=2$ with the expectations conditional on the information available at time 2 and so on until $k=N-1$.

\footnotetext{
${ }^{15}$ For comparison reasons, $D U A L P C$ uses the same procedure both in EOF and DUAL to update the estimate and the covariance of the parameters. This procedure is based on Kalman Filter. See, for instance Kendrick (1981, page 104), for details.
} 


\section{Computing the conditional expectations}

To compute the mean of the product of matrices appearing in the feedback rule, in the Riccati equations and in the optimal cost-to-go it is customary to exploit the fact that the Riccati matrices are not stochastic. When the parameters in $\mathbf{A}$ and $\mathbf{B}$ are assumed identically and independently distributed, it is possible to show that the mean of each element of the resulting matrix, say $\mathbf{R}$ with $\mathbf{R}=\mathbf{A}^{\prime} \mathbf{K B}$, takes the form ${ }^{16}$

$$
E\left(r_{i j}\right) \equiv E\left(\mathbf{a}_{i}^{\prime} \mathbf{K} \mathbf{b}_{j}\right)=E\left(\mathbf{a}_{i}^{\prime}\right) \mathbf{K} E\left(\mathbf{b}_{j}\right)+\operatorname{tr}\left[\mathbf{K} \boldsymbol{\Sigma}^{\mathbf{b}_{j} \mathbf{a}_{i}}\right]
$$

where $E\left(\mathbf{a}_{i}^{\prime}\right)$ is the mean of the elements appearing in the $i$-th row of matrix $\mathbf{A}^{\prime}$, or in the $i$-th column of matrix $\mathbf{A}, E\left(\mathbf{b}_{j}\right)$ the mean of the $j$-th column of $\mathbf{B}, \boldsymbol{\Sigma}^{\mathbf{b}_{j} \mathbf{a}_{i}}$ the covariance between the elements in $\mathbf{b}_{j}$ and $\mathbf{a}_{i}^{\prime}$ and $\operatorname{tr}[$.$] the trace$ operator. On the other hand if the parameters in $\mathbf{A}$ and $\mathbf{B}$ are modeled as in (5) and (6), the expectations needed to compute $\mathbf{K}_{N-1}$ at time 0 take the form $E_{0}\left(\mathbf{A}_{N-1}^{\prime} \mathbf{K}_{N} \mathbf{B}_{N-1}\right)$ and equation (36) is replaced by

$$
\begin{aligned}
E_{0}\left(r_{i j, N-1}\right) & \equiv E_{0}\left(\mathbf{a}_{i, N-1}^{\prime} \mathbf{K}_{N} \mathbf{b}_{j, N-1}\right) \\
& =E_{0}\left(\mathbf{a}_{i, N-1}^{\prime}\right) \mathbf{K}_{N} E_{0}\left(\mathbf{b}_{j, N-1}\right)+\operatorname{tr}\left[\mathbf{K}_{N} \boldsymbol{\Sigma}_{0, N-1}^{\mathbf{b}_{j} \mathbf{a}_{i}}\right]
\end{aligned}
$$

where $E_{0}\left(\mathbf{a}_{i, N-1}^{\prime}\right)$ is the mean of the elements appearing in the $i$-th row of matrix $\mathbf{A}_{N-1}^{\prime}$, or in the $i$-th column of matrix $\mathbf{A}$, conditional on the information on the parameters available at time $0, E_{0}\left(\mathbf{b}_{j, N-1}\right)$ the mean of the $j$-th column of $\mathbf{B}_{N-1}$ similarly defined, $\mathbf{K}_{N}=\mathbf{W}_{N}$ a deterministic matrix by assumption and $\boldsymbol{\Sigma}_{0, N-1}^{\mathbf{b}_{j} \mathbf{a}_{i}}$ is defined as

$$
\boldsymbol{\Sigma}_{0, N-1}^{\mathbf{b}_{j} \mathbf{a}_{i}} \equiv E_{0}\left\{\left[\mathbf{b}_{j, N-1}-E_{0}\left(\mathbf{b}_{j, N-1}\right)\right]\left[\mathbf{a}_{i, N-1}^{\prime}-E_{0}\left(\mathbf{a}_{i, N-1}^{\prime}\right)\right]\right\}
$$

The mean and variance of the rows and columns of $\mathbf{A}$ and $\mathbf{B}$ appearing in equation (37) and (38) have not been explicitly defined so far. However it is apparent that the $i$-th column of matrix $\mathbf{A}$ can be written as $\mathbf{S}_{i} \boldsymbol{\theta}$ with $\mathbf{S}_{i}$ a selecting matrix of dimension $n \times s$ defined as

$$
\mathbf{S}_{i}=\left[\begin{array}{llllll}
\mathbf{S}_{i, 1} & \ldots & \mathbf{S}_{i, n} & \mathbf{S}_{i, n+1} & \ldots & \mathbf{S}_{i, n+m} \\
\mathbf{S}_{i, n+m+1}
\end{array}\right]
$$

where the $\mathbf{S}_{i, j}$ block of dimension $n \times n$ is equal to the identity matrix if $i=j$ and the null matrix $\mathbf{O}$ otherwise. Then for $i$ going from 1 to $n, \mathbf{S}_{i}$ selects the elements of $\boldsymbol{\theta}$ corresponding to the $i$-th column of $\mathbf{A}$, for $i$ going from $n+1$ to $n+m$ it selects the $(i-n)$-th column of $\mathbf{B}$ and for $i=n+m+1$ it selects the parameters in $\mathbf{c}$.

\footnotetext{
${ }^{16}$ See, e.g., pp. 49-50 and Appendix B in Kendrick (1981, 2002).
} 
Equations (5) and (6) describe the behavior of all the parameters and can be used to compute the mean and variance of the parameters at time $N-1$, given the mean and variance of $\boldsymbol{\theta}_{0}$ at time 0 , namely ${ }^{17}$

$$
\begin{gathered}
E_{0}\left(\boldsymbol{\theta}_{N-1}\right)=\mathbf{D}^{N-1} E_{0}\left(\boldsymbol{\theta}_{0}\right)=\mathbf{D}^{N-1} \boldsymbol{\theta}_{0 \mid 0} \\
E_{0}\left\{\left[\boldsymbol{\theta}_{N-1}-E_{0}\left(\boldsymbol{\theta}_{N-1}\right)\right]\left[\boldsymbol{\theta}_{N-1}-E_{0}\left(\boldsymbol{\theta}_{N-1}\right)\right]^{\prime}\right\}= \\
\mathbf{D}^{N-1} \boldsymbol{\Sigma}_{0 \mid 0}^{\boldsymbol{\theta \theta}}\left(\mathbf{D}^{N-1}\right)^{\prime}+\mathbf{D}^{N-2} \boldsymbol{\Gamma}\left(\mathbf{D}^{N-2}\right)^{\prime}+\ldots+\boldsymbol{\Gamma}
\end{gathered}
$$

Then using the $\mathbf{S}_{i}$ matrix, the mean and variance of the individual columns of $\mathbf{A}$ and $\mathbf{B}$ can be promptly isolated, that is

$$
\begin{aligned}
& E_{0}\left(\mathbf{a}_{i, N-1}^{\prime}\right)=\mathbf{S}_{i} \mathbf{D}^{N-1} E_{0}\left(\boldsymbol{\theta}_{0}\right) \\
& E_{0}\left(\mathbf{b}_{j, N-1}^{\prime}\right)=\mathbf{S}_{n+j} \mathbf{D}^{N-1} E_{0}\left(\boldsymbol{\theta}_{0}\right)
\end{aligned}
$$

for $i=1, \ldots n$ and $j=1, \ldots, m$. Similarly equation (38) can be rewritten as

$$
\begin{aligned}
\boldsymbol{\Sigma}_{0, N-1}^{\mathbf{b}_{j} \mathbf{a}_{i}} & \equiv E_{0}\left\{\mathbf{S}_{n+j}\left[\boldsymbol{\theta}_{N-1}-\mathbf{D}^{N-1} E_{0}\left(\boldsymbol{\theta}_{0}\right)\right]\left[\boldsymbol{\theta}_{N-1}-\mathbf{D}^{N-1} E_{0}\left(\boldsymbol{\theta}_{0}\right)\right]^{\prime} \mathbf{S}_{i}^{\prime}\right\} \\
& =\mathbf{S}_{n+j}\left[\mathbf{D}^{N-1} \boldsymbol{\Sigma}_{0 \mid 0}^{\boldsymbol{\theta} \boldsymbol{\theta}}\left(\mathbf{D}^{N-1}\right)^{\prime}+\mathbf{D}^{N-2} \boldsymbol{\Gamma}\left(\mathbf{D}^{N-2}\right)^{\prime}+\ldots+\boldsymbol{\Gamma}\right] \mathbf{S}_{i}^{\prime}
\end{aligned}
$$

Again the role of the $\mathbf{S}$ matrix is to isolate, in this case from the $s \times s$ covariance matrix $\boldsymbol{\Sigma}^{\boldsymbol{\theta} \boldsymbol{\theta}}$ associated with the whole parameter vector $\boldsymbol{\theta}$, the $n \times n$ matrix of covariances associated with the parameters in the $i$-th column of $\mathbf{A}$ and the $j$-th column of $\mathbf{B}$.

At this point the Riccati matrix $\mathbf{K}_{N-1}$, and $\mathbf{p}_{N-1}$, can be computed. Both $\mathbf{K}_{N-1}$ and $\mathbf{p}_{N-1}$ are deterministic because they are functions of the means and variances of random variables. ${ }^{18}$. Therefore the procedure sketched in this section can be used to compute $\mathbf{K}_{N-2}$, and $\mathbf{p}_{N-2}$, and so on and so forth until $\mathbf{K}_{1}$, and $\mathbf{p}_{1}$, needed to compute the feedback rule for the control at time 0 .

\footnotetext{
${ }^{17}$ Equation (40) and (41) follow directly from (5). In the special case $N=3$, they look like

$E_{0}\left(\boldsymbol{\theta}_{3-1}\right)=\mathbf{D}\left(\mathbf{D} \boldsymbol{\theta}_{0 \mid 0}\right)=\mathbf{D}^{2} \boldsymbol{\theta}_{0 \mid 0}$

$E_{0}\left\{\left[\boldsymbol{\theta}_{2}-E_{0}\left(\boldsymbol{\theta}_{2}\right)\right]\left[\boldsymbol{\theta}_{2}-E_{0}\left(\boldsymbol{\theta}_{2}\right)\right]^{\prime}\right\}=\mathbf{D}^{2} \boldsymbol{\Sigma}_{0 \mid 0}^{\boldsymbol{\theta} \boldsymbol{\theta}}\left(\mathbf{D}^{2}\right)^{\prime}+\mathbf{D} \boldsymbol{\Gamma} \mathbf{D}^{\prime}+\boldsymbol{\Gamma}$.

${ }^{18}$ See, e.g., Hamilton 1994, Section 4.1, footnote 1)
} 


\section{The Beck and Wieland model}

In this section we will see that the Beck and Wieland (2002) model can be cast into the above framework. Furthermore we will show that, when the parameters are as in Beck and Wieland (2002) and Amman et al. (2008), this model is a special case and the optimal control is identical to that obtained following the presentation of Kendrick (1981, 2002, Chapter 6 and 7).

Following Beck and Wieland (2002) the decision maker is faced with a scalar linear stochastic optimization problem of the form ${ }^{19}$

$$
\begin{aligned}
\underset{\left[u_{k}\right]_{k=0}^{N-1}}{\operatorname{Min}} E\left[\delta^{N}\left(x_{N}-\hat{x}_{N}\right)^{2}+\right. \\
\\
\left.\quad \sum_{k=0}^{N-1} \delta^{k}\left\{\left(x_{k}-\hat{x}_{k}\right)^{2}+\omega\left(u_{k}-\hat{u}_{k}\right)^{2}\right\} \mid\left(x_{0}, b_{0}, v_{0}^{b}\right)\right]
\end{aligned}
$$

subject to the equations

$$
\begin{aligned}
x_{k+1} & =\alpha+\beta_{k} u_{k}+\gamma x_{k}+\epsilon_{k} \\
\beta_{k+1} & =\beta_{k}+\zeta_{k}
\end{aligned}
$$

where $\delta$ is a discount factor, $\hat{x}$ and $\hat{u}$ denote the desired value of the state and control, respectively, $\epsilon_{k} \sim N\left(0, \sigma_{\epsilon}^{2}\right)$ and $\zeta_{k} \sim N\left(0, \sigma_{\zeta}^{2}\right)$. It is assumed that $x_{0}$ is given and the model contains one uncertain parameter $\beta$, with an initial estimate of its value at $k=0, E_{0}\left(\beta_{0}\right)=b_{0}$, and an initial estimate of its variance at time $k=0, V A R_{0}\left(\beta_{0}\right)=v_{0}^{b}$. The parameters $\alpha$ and $\gamma$ are constant. Beck and Wieland assume in their paper that $N \rightarrow \infty$. In contrast we will assume that the planning horizon is finite, hence $N<\infty$. Furthermore, we have adopted the timing convention from Kendrick (1981, 2002) where the control, $u_{k}$, has a lagged response on the state, $x_{k}$. Moreover the desired path for the state and the control, and the penalty weight on the latter, is zero.

With this set of assumptions, the above model can be fitted with little effort into the format of equations (2)-(8) when $\mathbf{A}_{k}=\gamma, \mathbf{B}_{k}=\beta_{k}, \mathbf{c}_{k}=\alpha$, $\mathbf{v}_{k}=\epsilon_{k}$ and

$$
\boldsymbol{\theta}_{k}=\left[\begin{array}{l}
\gamma \\
\beta_{k} \\
\alpha
\end{array}\right], \quad \boldsymbol{\eta}_{k}=\left[\begin{array}{l}
0 \\
\zeta_{k} \\
0
\end{array}\right]
$$

\footnotetext{
${ }^{19}$ In an earlier strand of literature, going back to the early Seventies, a similar model and approach is discussed. See, MacRae $(1972,1975)$.
} 
and $\mathbf{D}$ is an identity matrix. In this case the covariance matrices are $\mathbf{Q} \equiv \sigma_{\epsilon}^{2}$ and

$$
\boldsymbol{\Gamma}=\left[\begin{array}{lll}
0 & 0 & 0 \\
0 & \sigma_{\zeta}^{2} & 0 \\
0 & 0 & 0
\end{array}\right]
$$

Furthermore it is assumed that $\mathbf{x}_{0}$ is given and

$$
\boldsymbol{\theta}_{0 \mid 0}=\left[\begin{array}{c}
\gamma \\
b_{0} \\
\alpha
\end{array}\right], \quad \boldsymbol{\Sigma}_{0 \mid 0}^{\boldsymbol{\theta} \boldsymbol{\theta}}=\left[\begin{array}{ccc}
0 & 0 & 0 \\
0 & v_{0}^{b} & 0 \\
0 & 0 & 0
\end{array}\right]
$$

In this case the only relevant $\mathbf{S}_{i}$ is $\mathbf{S}_{2}=\left[\begin{array}{lll}0 & 1 & 0\end{array}\right]$ and the optimal cost can be expressed as

$$
J_{j}^{*}=\frac{1}{2} \mathbf{x}_{j}^{\prime} \mathbf{K}_{j} \mathbf{x}_{j}
$$

because the desired paths for the state and control are $0, \alpha=0$, $\omega=\boldsymbol{\Lambda}_{j}=0$ and $\mathbf{F}_{j}=0$. The optimal control at time 0 is

$$
\mathbf{u}_{0}^{*}=\mathbf{G}_{0} \mathbf{x}_{0}
$$

where

$$
\begin{aligned}
\mathbf{G}_{0} & =-\left[E_{0}\left(\mathbf{B}_{0}^{\prime} \mathbf{K}_{1} \mathbf{B}_{0}\right)\right]^{-1} E_{0}\left(\mathbf{B}_{0}^{\prime} \mathbf{K}_{1} \mathbf{A}_{0}\right) \\
& =-\left[\mathbf{K}_{1}\left(b_{0}^{2}+v_{0}^{b}\right)\right]^{-1}\left[\mathbf{K}_{1} \gamma b_{0}\right]=-\left(b_{0}^{2}+v_{0}^{b}\right)^{-1} \gamma b_{0}
\end{aligned}
$$

This means that the optimal expected feedback control, or myopic cautionary rule as in Beck and Wieland (2002), is solely a function of the current information about the stochastic parameter. There is no need to compute future expectations as the Riccati matrix does not enter the feedback matrix. Hence, in the Beck and Wieland (2002) case, the time varying parameter solution can be obtained using the framework of Kendrick (1981, 2002, Chapter 6 and 7) and equation (50) does not need to be approximated.

\section{Summary}

In this paper we derived the closed loop form of the Expected Optimal Feedback rule with time varying parameters. As such this paper extends the work of Kendrick. Furthermore, we showed that the Beck and Wieland model can be cast into this framework and basically can be treated as a special case of this solution. 


\section{References}

Amman, H. M. and Kendrick, D. A.: 2001, Open loop feedback with timevarying parameters: Derivations, Working paper, Department of Economics, University of Texas, Austin, Texas 78712, USA.

Amman, H. M., Kendrick, D. A. and Tucci, M. P.: 2008, Solving the Beck and Wieland model with optimal experimentation in DUALPC, $A u$ tomatica 44, 1504-1510.

Anderson, B. D. O. and Moore, J. B.: 1979, Optimal Filtering, Prentice Hall, Englewood Cliffs, USA.

Beck, G. and Wieland, V.: 2002, Learning and control in a changing economic environment, Journal of Economic Dynamics and Control 26, 1359-1377.

Bertsekas, D. P.: 2005, Dynamic Programming and Stochastic Control, Vol. 1, third edn, Athena Scientific, Boston, USA.

Chow, G. C.: 1973, Effect of uncertainty on optimal control policies, International Economic Review 14(3), 632-645.

URL: http://www.jstor.org/stable/2525976? origin =JSTOR-pdf

Chow, G. C.: 1975a, Analysis and control of dynamic economic systems, John Wiley, New York, New York.

Chow, G. C.: 1975b, A solution to optimal control of linear systems with unknown parameters, Review of Economics and Statistics 57(3), 338-345. URL: http://www.jstor.org/stable/1923918?origin=JSTOR-pdf

Granger, C. W. J.: 2008, Non-linear models: Where do we go next - timevarying parameter models?, Studies in Nonlinear Dynamics and Econometrics 12, 1-11.

Hamilton, J. D.: 1994, Time Series Analysis, Princeton University Press, Princeton, USA.

Harvey, A.: 1981, Time series models, Philip Allan, Oxford, UK.

Johnston, J.: 1984, Econometric Methods, third edn, McGraw-Hill.

Kendrick, D. A.: 1981, Stochastic control for economic models, first edn, McGraw-Hill Book Company, New York, New York, USA. See also Kendrick (2002).

Kendrick, D. A.: 2002, Stochastic control for economic models. University of Texas.

URL: http://www.utexas.edu/cola/depts/economics/faculty/dak2 
Kendrick, D. A. and Majors, J.: 1974, Stochastic control with uncertain macroeconomic parameters, Automatica 10, 587-594.

MacRae, E. C.: 1972, Linear decision with experimentation, Annals of Economic and Social Measurement 1, 437-448.

MacRae, E. C.: 1975, An adaptive learning role for multiperiod decision problems, Econometrica 43, 893-906.

Tucci, M. P.: 1989, Time-varying Parameters in Adaptive Control, PhD thesis, Department of Economics, University of Texas, Austin, Texas 78712, USA.

Tucci, M. P.: 1997, Adaptive control in the presence of time-varying parameters, Journal of Economic Dynamics and Control 22, 39-47.

Tucci, M. P.: 2004, The Rational Expectation Hypothesis, Time-varying Parameters and Adaptive Control, Springer, Dordrecht, the Netherlands.

Turnovsky, S. J.: 1975, Optimal choise of monetary instruments in a linear economic model with stochastic coefficients, Journal of Money, Credit and Banking 7(1), 51-80.

URL: http://www.jstor.org/stable/1991252?origin=JSTOR-pdf

Turnovsky, S. J.: 1976, Optimal stabilization policies for stochastic linear systems: The case of correlated multiplicative and additive disturbances, Review of Economics and Statistics 43(1), 191-194.

URL: http://www.jstor.org/stable/2296614?origin=JSTOR-pdf

Turnovsky, S. J.: 1977, Optimal control of linear systems with stochastic coefficients and additive disturbances, in J. Pitchford and S. J. Turnovsky (eds), Application of Control Theory to Economic Analysis, North-Holland Publishers (Elsevier), Amsterdam, the Netherlands, chapter 11, p. 363. 